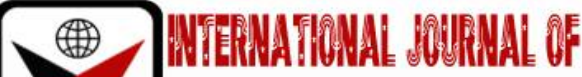

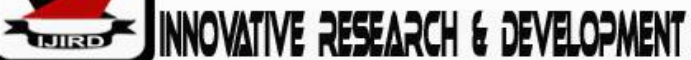

ISSN 2278 - 0211 (Online)

\section{Colour Consistency in Print: Ghanaian Printers' Knowledge and Awareness of Colour Management}

Francis Kofi Nimo Nunoo
Lecturer, Department of Publishing Studies of the Kwame Nkrumah,
University of Science and Technology, Kumasi, Ghana
Eric Anane-Antwi
Lecturer, Department of Publishing Studies of the Kwame Nkrumah,
University of Science and Technology, Ghana
Ismaila Moro
Lecturer Department of Communication Design, KNUST, Ghana
Isaac Kofi Appiah
Lecturer, Department of Publishing Studies of the Kwame Nkrumah,
University of Science and Technology, Ghana
Emmanuel Winston Churcher
Production Planner, Buckpress Gh. Ltd, a Leading Printing
Company in Accra, Ghana

\begin{abstract}
:
With the advances print industry, high-quality printout is crucial for the survival of any press house as the creation and retention of loyal customers greatly depend on it. However, the ability to consistently print high-quality materials is one of the greatest challenges in the printing industry over the years due to differences and limitations in various imaging devices as well as software used in colour reproduction workflows. Colour Management system has proven to be the surest way to achieve consistent colour reproductions. However, understanding how devices capture and reproduce colour, as well as, ensuring an accurate, predictable and consistent reproduction of colour requires a change in workflow and efficient utilisation of digital technologies available. In spite of the use of colour management system and available print technologies the ability to print consistent high-quality colour reproduction repeatedly still remains a great challenge for less endowed press houses all over the world. This study employed the survey research design making use of questionnaires and observation to ascertain the awareness and knowledge levels of print technicians on colour management issues in selected print houses in Ghana. The findings revealed that many of the presses have the required imaging devices yet not calibrated, environmental conditions required for effective calibration were not strictly adhered to and there is low level of awareness on the use of colour management system. The study concludes that the sampled press houses still have fair knowledge about effective colour management practices and the use of available print technologies that help presses to consistently produce high-quality prints. The researchers, therefore, recommend that the presses adhere to effective colour management practices and also employ the available print technologies to help improve their colour output consistency and quality to stay competitive.
\end{abstract}

Keywords: Colour Management System, Profiling, Calibration, digital technologies

\section{Introduction}

Traditionally, printing was the only output for spreading messages and information to the masses when referring to non-personal communication channels (Kotler, Armstrong, Harris, \& Piercy, 2013). The advent of radio and television as well as digital technologies such as the internet has democratised the stranglehold of the printing technology on information content delivery. Print in general is now seen as just an output channel among others for those who are trying to reach an audience(Mejtoft, 2006). Although the electronic media market is in rapid development, evidence show many people prefer reading on paper rather than on displays(Nussbaum, 2010). This is a very strong motivation to printing presses as it affirms that the printing business will continue to be in existence in spite of the advances in the electronic delivery of information content. On the other hand, it informs printing presses that competition is no longer with other printing presses but also with the electronic media market.

Every company active in an industry is affected by its surroundings, which in turn makes up the competitive environment for the industry, the printing industry is no exception. The demand for a high-quality printout is crucial for the survival of a press house and the creation of loyal customers. The fast-technological development in the printing and, 
also, in many parts of the graphic arts industry, has introduced new possibilities which has further made the competition in the industry more dynamic and intense.

Since the beginning of printing, the reproduction of continuous tone art has posed a great challenge to graphic artists and printers. The transformation of photographic originals into realistic and compelling printed images is an arduous process, especially with traditional printing technologies like Offset Lithography(Agfa-Gavaert, 1997). This challenge is as a result of colour or coloured images not showing the same resolution on different input and output devices, such as; scanners, cameras, monitors, printers. That is to say, just as colour is interpreted differently by individuals, so is colour differently displayed and reproduced by different electronic devices. Even with the "closed-ended" colour imaging systems and the specialised skills required by printing in the earlier print era, there were obvious colour failures noted by unhappy customers which led to cases of rejection of works and ultimately loss of money and potentially loyal customer (Green, 2010). The advent and dominance of digital photographic imaging devices has led to the era of "open" colour imaging systems.

Today, there are several colour spaces (sRGB, RGB 64, Adobe RGB, etc.), and a vast array of printers (electrophotographic, ink jet, dye thermal transfer, etc.), each with its own colorants and recommended substrate for good print outputs, have made the ability to control colour quality a challenge, if not a nightmare.

High qualitycolour print will enable printing presses satisfy their customers' needs while increasing profits. This is due to the fact that colour output in a printed work is a factor that greatly affects a customer's buying decision. In the end, colour management is what makes possible this feat. Due to the role colour management plays in ensuring quality output as regards colour reproduction, it is now imperative for printing presses to employ the tools and applications colour management systems offer so as to keep clients satisfied and attain their goodwill. However, understanding how devices capture and reproduce colour, as well as, ensuring an accurate, predictable and consistent reproduction of colour requires a change in workflow and efficient utilisation of digital technologies(Adams II \& Weisberg, 2000; Ashe, 2014; Enoksson, 2010). There are various types of equipment (i.e. printing machinery) and many software applications (i.e. image processing, profile making) on the market with different characteristics and algorithms, each rendering colours differently. This generate certain practical challenges as images move through the various printing workflows (Enoksson, 2010). These challenges can be mitigated with effective colour management systems.

Effective colour management system employs the use of advanced applications which allow colour to be reproduced in a similar manner on different displays and print platforms (Ploumidis, 2008).

In the graphic arts and printing industry, colour measuring instruments are used primarily for determining the colour characteristics of objects, such as prints and proofs, and for measuring the behaviour of input and output devices. Successful reproduction of colour images depend on a number of factors; starting with the characteristics of the original photograph, the scanning process, colour correction, halftoning, and printing processes with each having their own effects on the outcome (Agfa-Gavaert, 1997).

In light of these factors, device calibration and characterization are very important tasks in determining the appropriate parameters and verifying the behaviour of the devices in use. In photography and printing, three main devices are used for measuring colour on monitors and printed output that is; densitometers, colorimeters, and spectrophotometers.

Colour management translates colour between devices using a device-independent Profile Connection Space (PCS) and standard profiles for each of the devices. A profile characterizes a device's colour reproduction capabilities (Adams II \& Weisberg, 2000). These management systems rely on International Colour Consortium (ICC) profiles to create the basis for colour conversions. The profiles are file formats which show gamut of a device (Köse, Şahinbaşkan, \& Güler, 2009). While colour proofs were obtained previously from prepared film or pre-prints made directly on a printing machine; today, since print preparation is achieved directly in a digital setting, proofs are now obtained from digital printers. With the use of the colour management system, digital proofs imitate the print outcomes. Through the ICCprofile, various colours and hues can be interpreted in a similar fashion regardless of the platform and application (computer type, monitor model, system construction and pre-press programmes), The ICC-format enables the colour space of a colour unit to be determined from a large number of measured values, and thus enables, for example, optimization of printing simulations, by using colour engines or colour profiling(Enoksson, 2010).

There has been significant growth of the printing industry in Ghana since its inception in the 1960's with the establishment of Ghana Printing Press, which later became Ghana Publishing Corporation(Prempeh, 2011). The print industry could be said to be one of the technologically advancing areas in the manufacturing sector in Ghana especially in the area of machinery.

In spite of these technological advancements, the quality of colour print has not seen much improvement as evident in the off-shoring of print services from the far east and south Africa. These improvements have been seen mainly in the design and print machinery. Nonetheless, less attention is given to workstation environment, abiding by print standards and creating required profiles as well as the required skilled personnel with requisite knowledge on colour reproduction. Ploumidis in 2008, opined that an educated workforce is a crucial prerequisite for high quality output in print production. According to him, a skilled craftsman is more reliable than any system or piece of machinery because decision-making takes place at every step of the colour management process and it can only be efficient if each individual has the requisite technical knowledge of the process.

The focus of this study is to ascertain the awareness and knowledge levels of print technicians on colour management implementation in selected printed houses in Ghana. 


\section{Methodology}

This was a descriptive study that used questionnaire and observation as data gathering tools. The study was done to ascertain the level of awareness and understanding of technicians of press houses on the subject of colour management. The study was conducted in major printing houses, from digital to typical offset lithography, in the Accra and Kumasi metropolises since they are the main hubs for printing activities in Ghana. A total of 40 press houses comprising of 20 from each of the two metropolises, were sampled. The purposive sampling technique was employed as the selection of these presses were based on preliminary studies by researchers on the volume and type of works, they produced and how active their pre-press sections were.

Questionnaire was developed based on the literature reviewed and was structured in two parts. Section one dealt with knowledge and practices relating to device calibration and profiling aimed at testing respondents' knowledge on these matters and how they impact on predictability and consistency of colour reproduction. Section two focused on pre-press technicians' response to colour separation and related issues that impact print quality. The overall response rate was 87.5\%, with $100 \%$ response from presses in Kumasi and 75\% from Accra.

\section{Findings}

\subsection{Device Availability}

Devices such as monitors, scanners, imagesetters, platesetters and laser printers must be available in order to make the Graphic Art workflow possible. As a result, the study sought to find out the type of devices being used in these presses and the responses is shown in Table 1 below. Those without important equipment like imagesetters or platesetters explained that they outsource for such services when the art work is done.

\begin{tabular}{|c|c|}
\hline Device Type & Number of Respondents \\
\hline Monitors & 35 \\
\hline Scanners & 34 \\
\hline Digital Cameras & 16 \\
\hline Imagesetters & 20 \\
\hline Platesetter (CTP) & 9 \\
\hline A3 size Laser Printers & 26 \\
\hline A3 size Inkjets & 18 \\
\hline
\end{tabular}

Table 1: Devices Used in the Press Houses

\subsection{Knowledge on Calibration and Profiling}

Many output devices performance capabilities change over time due to phosphor instability (especially in monitors), changes in colorants and room humidity (Köse et al., 2009). Calibrating the devices is a basic solution to mitigate these challenges (Upton, 2001). Respondents were asked whether they calibrate their devices and how often. Their responses are shown in figures 1 and 2. Interval for calibration and recalibration depends largely on the stability of devices (with regards to accuracy) and how often the device is used, although a minimum of once every two weeks is recommended (Upton, 2001).

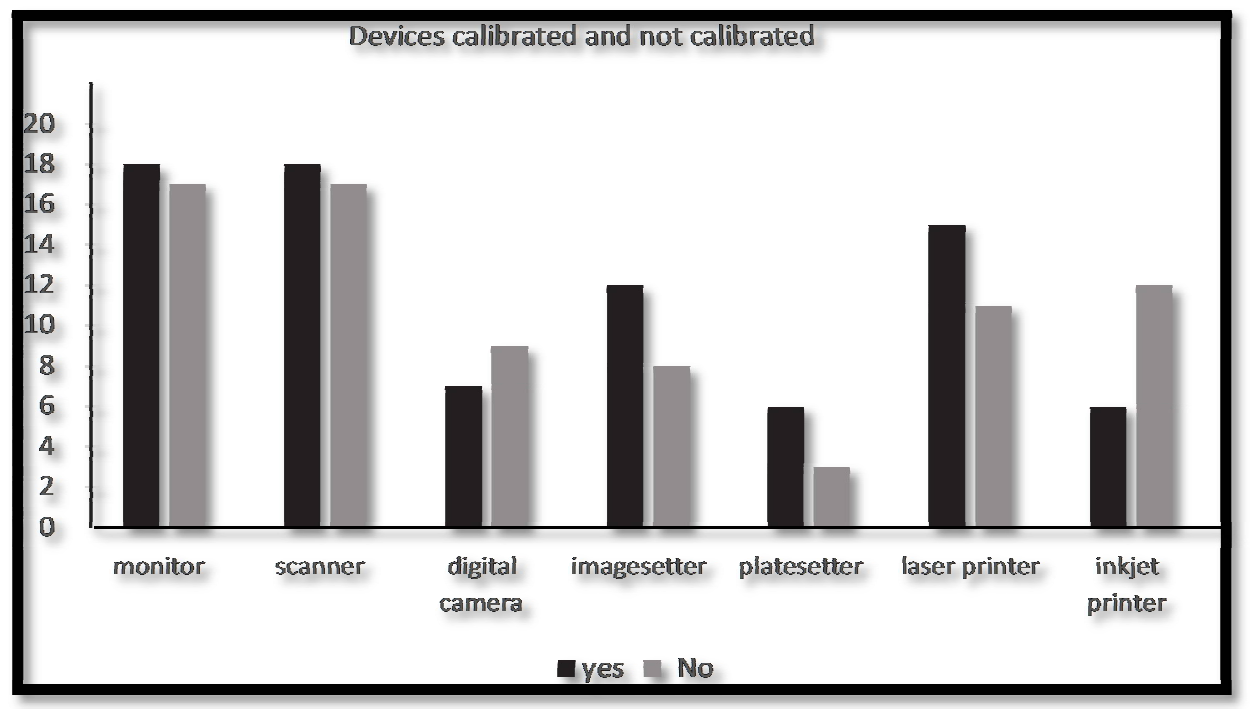

Figure 1: Responses on Calibration and Non-Calibration of Devices 


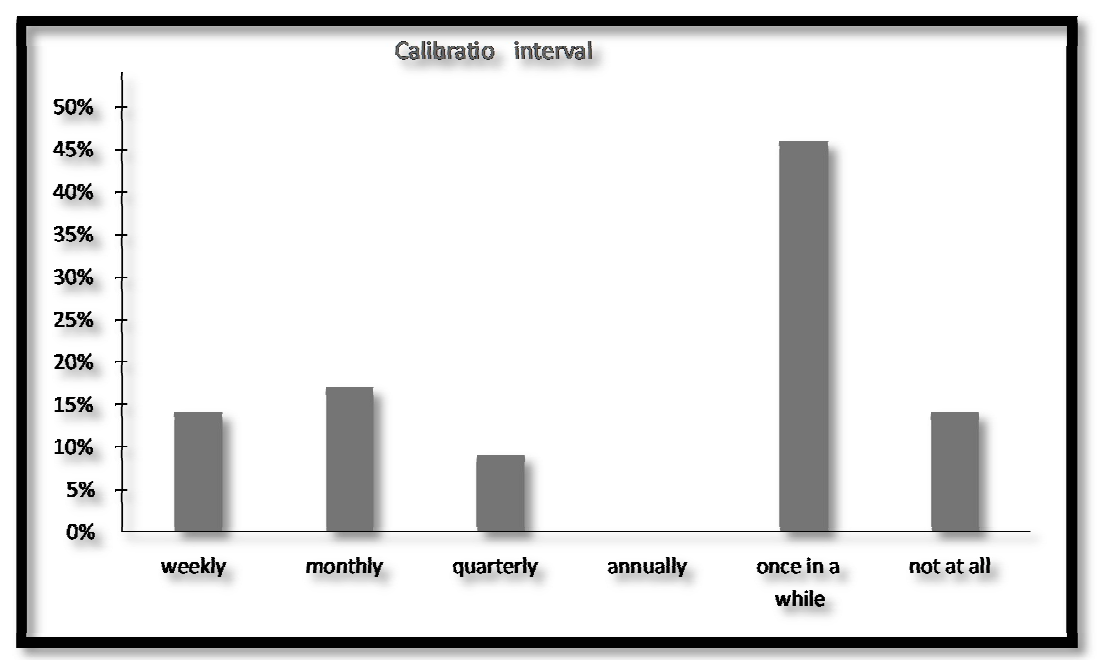

Figure 2: Calibration in Intervals

As regards knowledge on colour profile, 25 press technicians responded positive with 10 answering no. Regarding how respondents calibrate their devices, majority (68.6\%) relied on visual evaluation (use of the eye), $20 \%$ used colorimeter, $8.6 \%$ and $3 \%$ densitometer and spectrophotometer respectively. The usage of colour measurement devices for calibration has a vast advantage over using visual valuation. This is due to the fact that each type of colour measurement instrument does something that the eye cannot do; for instance, assigning a specific value to the colour that can be consistently analysed in terms of numeric tolerances and control limits.

Aside using measuring instruments and having a sound working environment condition(with grey pattern studio walls, avoidanceof brightly-coloured artwork and curtains hanging adjacent to monitorsand monitors notpositioned near windows or room lighting that is glaring or changes frequently), monitors should be shielded on top and at the sides with a cardboard or hood and their brightness and contrast knobs set at the desired levels (Ashe, 2014)are fundamental in calibrating devices and viewing outputs. Per the data analysed, it was observed that most of these conditions required for effective calibration were not strictly adhered to.

Although majority of the respondents $(60 \%)$ were of the view that their working environments had no effect on their displays and their pre-press output, their environments served as viable source for metameric occurrence because many of these did not have the D50 requirement for achieving high quality output.

Regardless of which device one is working with, colour management involves two stages; calibration, and characterization or profiling. In spite of the fact that devices used in the graphics art industry are profiled by manufacturers, it becomes imperative that custom profiles be created. A custom profile takes into consideration the colour measurement devices' (spectrophotometer, densitometer, colorimeter) values used for profiling a particular unit (monitor, scanner, plate setter and printer) as well as the numerous colour profiles for intended substrates based on the various printed standards (Waite, 2006). Although $71 \%$ of respondents indicated that they knew what colour profiles were and the relevance of costumed profiles for quality print, data analysed showed that $97 \%$ of the press houses surveyed worked with sheetfed machines suggesting that the specifications for colour profile to be used must be sheetfedbased but responses proved otherwise. For coated stocks, 58.7\% of respondents answered that they used the default specification which is the US Web Coated (SWOP) V2 and also the US Web Uncoated V2 which are meant for web printing machines. For uncoated stocks, $42.3 \%$ indicated that they use the correct specification which is the US Sheetfed Uncoated V2 but the remaining $57.6 \%$ chose the wrong specifications. From these indications, it can be said that profiles would be problematic in the work of respondents but 50\% responded that they encountered problems with profiles only occasionally with only $3.8 \%$ indicating that they often encountered problems with profiles. These indications from respondents show that they know very little about profiles and their effect on pre-press output.

On the question of what profile do the press house select for their various substrate jobs, $75 \%$ of the respondents left that part of the questionnaire unanswered. These responses revealed that although respondents indicated that they knew what profiles were, further enquiries denied this fact. From the analyses, it could be deduced that majority of presses in Ghana knew very little of what profiles were and therefore inference can be made that awareness in colour management in Ghanaian printing presses is low.

\subsection{Knowledge on Colour Separation and Related Issues (Application)}

This section of the questionnaire was to test the practices and cautions observed by the presses surveyed in their work before putting their work finally in print. The questionnaire first asked respondents the colour space they save their works in before colour separation. Options of RGB and CMYK were given for the above question. After the analysis, it was observed that majority (86\%) chose CMYK for their works before separation. This goes to say that only a minority (14\%) prepared their work in RGB for separation.

Since presses commonly print in the four process colours (sometimes with one or more spot colours), and the existence of other related print problems such as misregistration and dot gain, it is advisable to save works for colour separation in CMYK so that four or more films and plates will be produced for printing. This ensures that continuous tone 
outputs match with their soft or hard proof copies. The fact that majority of the presses choose CMYK process colours shows that they are not ignorant of this fact.

An enquiry into which colour space (RGB, CMYK, Adobe RGB 98, sRGB etc.) respondents usually use when working on images yielded $65.7 \%$ of the responses choosing CMYK. The reason for the higher option in CMYK was for the purpose of playing safe when editing images on monitors (this prevents having to change the colour space to CMYK before colour separation). This reason, though sensible, has its shortcomings since the gamut for CMYK is relatively smaller than the other colour spaces. Printers and their CMYK colorants, in general, have limited gamut than most monitors (RGB, sRGB, Adobe 98 etc.).

Regarding the use of RGB, 34.3\% respondents responded affirmative before converting to CMYK if the work will ultimately be printed. This according to them yielded desirable results. An indication from the analysis made the researchers aware that a few of the respondents knew about other colour spaces (sRGB and Adobe RGB) aside these primary colour spaces (CMYK and RGB).

The relationship between the image setter / plate setter resolution (dot per inch (dpi)) and the screen ruling (lines per inch (lpi)) determines the halftone quality of the printed output. The screen ruling used for a job depends on the resolution of the plate setter as well as the paper stock and type of press to be used. In a bid to ascertain the resolution chosen for coated stock (e.g. Chromocoat, Art Paper, etc.) and uncoated stock (Newsprint, Bond, etc.) a range between $100 \mathrm{dpi}-300 \mathrm{dpi}$ and $72 \mathrm{dpi}-100 \mathrm{dpi}$ respectively were given to respondents to choose for each stock they print on.

It is advisable that full-colour work on newsprint (uncoated stock) should be commonly printed using a screen ruling of 100 lpi because newsprint absorbs a great deal of ink and the presses operate at high speed. Majority (71.4\%) of respondents, however, chose between 100dpi-300dpi for the resolution they used on uncoated stock. This choice would lead to over saturation of colour on the newsprint or bond paper and make the images look muddy as expressed by some of the operators. The remaining $28.6 \%$ chose $72 \mathrm{dpi}-100 \mathrm{dpi}$. Their reason was that a higher resolution makes printing difficult to control and image rendering undesirable in the final output.

The thickness of paper (in grams) and its special characteristics influence the choice of resolution. Full-colour magazines on coated paper are usually printed with a screen ruling of $133 \mathrm{lpi}$, whereas sheet-fed and commercial printing is typically done at 150 lpi (Waite, 2006). 80\% of the respondents correctly chose resolution between $100 \mathrm{dpi}-300 \mathrm{dpi}$ on coated stock. The few, $20 \%$ who chose $72 \mathrm{dpi}-100 \mathrm{dpi}$ seemed ignorant on the impact of resolution on the quality of a coloured print.

ICC-Profiling has afforded operators with enough options where rendering intents are concerned. The rendering intents are basically four; Relative Colorimetric, Absolute Colorimetric, Perceptual and Saturation. Each of these rendering intents has its type of graphic images they are applied to and is also in part based on the preference of the operator. In the survey to know which intent was normally employed, relative Colorimetric had the highest response rate of $69.4 \%$, perceptual and absolute Colorimetric each being $11.1 \%$ and Saturation being $8.3 \%$.

One of the reasons why relative colorimetric scored high was because many printed line copies come with little graphics in them. Again, this option was also set at default in most dialogue boxes of imaging software and so was left untouched. In most cases, the researchers had to open the dialog box with rendering intent options since some respondents knew virtually nothing about it.

The aim of colour management is to ensure consistency, repeatability and predictability in coloured works. Respondents were asked if they apply colour management systems in their works and the challenges, they encountered during the implementation of colour management. They were also asked to grade their output after pre-press especially with regards to colour consistency, repeatability and predictability.

$74 \%$ responded in the affirmative. This score is doubtful taking into consideration how majority of them failed to undertake calibration and also how they knew very little about profiling. The range of resolution most of them use for their coated and uncoated works also buttresses the fact that they do not implement colour management systems in their workflow.

As regards problems they encountered with the implementation of colour management, 15 respondents answered that they encountered no problems at all,22 occasionally andonly 3stated that they encounter persistent challenges. Although many of the responds have expressed the notion of not having challenges in colour management implementation, contrasting it with their earlier responses on calibration and other colour management practices, this cannot be taken as the truth. As a result, these presses could encounter some significant problems in their 'supposed' implementation of colour management systems.

When respondents were asked to grade their works output after pre-press, $28 \%$ graded theirs as excellent, $46 \%$ graded theirs as very good with $26 \%$ grading theirs as good. Achieving an excellent and very good output is questionable, in that respondents' failure to implement a strict colour-managed work flow would always result in variations in pre-press output. This would result in the onscreen output mismatching with the actual press output. Their grading of output as good can be as a result of what is commonly considered by printers as "the achievable" or "expected" output of a printed work in Ghana.

\section{Discussion on Findings}

From the analysis of the findings, it is incontestable that the awareness of colour management is low even though there is the appreciation of the colour problem in their operations. Design, graphics and imaging professionals are aware of the fact that colour is a very important part of the consumer's buying decision (X-Rite, 1998). This has a direct consequence on their competitive edge and adherence to colour fidelity. At the beginning of the printing process the consistent reproduction of the designers' ideas expressed in a digital format or otherwise and the customers' specification 
come in various forms - as a digital file, on a plate or an already printed document. The printer might also be given data in the form of a written or oral specification.

From the study it was observed that majority of respondents went about their pre-press activities with little or no attention to the implementation of colour management in their workflow. Devices such as monitors, scanners, imagesetters, platesetters and laser printers must be available in order to make the graphic art workflow possible (X-Rite, 1998), most the studied presses had these devices, however, presses without some of these devices such as imagesetters and plate setters out sourced their colour separation and plate burning activities. It has been observed that outsourcing some of these tasks bring about loss of control over the pre-press process due to differences in imaging devices. The conception of a print product begins from the prepress. An uncontrolled and strict adherence to standards and specifications of a client could cause failure in the print project from the outset. Process controls are the key factors for successful colour management. A process which is not consistent and repeatable will render a colour profile useless(Marin, 2004).

\subsection{Knowledge on Calibration}

The few respondents who paid some attention to colour management focused on some parts and left other parts out. This was as a result of their deficiency in the machines available for the execution of a printed job. The devices used in the print workflow such as output devices could have their performance capabilities change over time due to phosphor instability (for monitors) and changes in colorants and room humidity which can throw their performance off course (XRite, 1998). The data suggested that reasonable attention was paid to calibration leaving out profiling. Regardless of which device one is working with, colour management always has two stages: calibration, and characterization or profiling (Ashe, 2014). Accuracy, predictability and consistency in a particular printed product could therefore be compromised.

Interval for calibration and recalibration depends largely on the stability of devices (with regards to accuracy) and how often the device is used, although a minimum of once every two weeks is recommended(Upton, 2001). In the cases of the presses studied, majority of them calibrated their devices once in a while and was observed that many of these devices had aging problem and therefore would require regular calibration. Aside the aging of devices, one other factor underlying the less cognisance for calibration and profiling could be blamed on the unavailability of colour measuring instruments in these press houses. In photography and printing, the three main devices used for measuring colour on monitors and printed output are densitometers, colorimeters, and spectrophotometers (Ashe, 2014). In the absence of these instruments, visual valuation (i.e. the use of the eye) is the main instrument used for calibrating devices. This was the norm for many of the presses studied. The usage of colour measurement devices for calibration has a vast advantage over visual valuation. This is due to the fact that each type of colour measurement instrument does something that the eye cannot do, e.g. assign a specific value to the colour that can be consistently analysed in terms of numeric tolerances and control limits(X-Rite, 1998). A subjective valuation (human eye) of the devices cannot guarantee a consistent, repeatable and accurate reproduction of colour.

Ensuring a good working environment is very necessary for calibration. Although majority of the respondents for this study had a reasonable awareness on the effects of their work environment on their displays and their pre-press output, their workstations served as viable areas for the occurrence of metamerism judging from the fact that they had no standard for their light intensity, as prescribed by CIE, to enable them adjust their lighting to match with where the customer would finally view their works. The Commission Internationale de l'Eclairage (the International Commission on Illumination), or CIE has established standards for illuminants or light sources. CIE Standard Illuminants or light sources include: D50, D55, D65, A, and F1 through F12. D50, D55 and D65 are daylight 5000, 5500 and 6500 Kelvin illuminants, respectively. CIE Standard Illuminant A is a tungsten light source. F1 through F12 are different standard fluorescent light sources (Ashe, 2014). The respondents could not give details as to the intensity of the light sources they use at their workstation. They basically sought out lamps or bulbs subject to the own preference. This could put their calibration and profiling at a disadvantage since the light has a direct influence on these processes.

While many manufacturers of devices used in the graphic art workflow are always profiled at source, it is advisable that custom profiles (source and destination profiles) be created for both input and output devices. A custom profile takes into consideration the colour measurement devices' (spectrophotometer, densitometer, and colorimeter) values used for profiling that particular unit (monitor, scanner, plate setter and printer) as well as the numerous colour profiles in imaging software. Many of the presses admitted to not knowing what profiles were, partly explaining why they did not have appropriate colour measuring tools. A check on their profile settings also revealed that they were stuck with the default settings that come with the devices and other proprietary software. Also, some of the respondents perceived profiles to be colour spaces (RGB and CMYK). The reliance on the default settings of devices and imaging software (CorelDraw, Adobe Photoshop, Adobe InDesign, etc.) meant that some printed works had been done with the wrong profiles. This was realised with most of them admitting to facing colour problems with the works they produced. Overall, knowledge on calibration could be considered as being low even though they appreciated the colour problem faced.

\subsection{Knowledge on Colour Separation and Related Issues}

This section of the questionnaire was to test the practices and cautions observed by presses in their works before printing. It was observed from the survey that most presses presented their works in CMYK for colour separation. This helped them avoid the uncharacteristic problems they may face during separation. Four plates or more are needed for a continuous tone reproduction especially by offset printers. On the same note, most designers gave the indication that they best work in the CMYK colour space than the RGB. They considered it safer since they will eventually come out in print. Very few worked in the RGB mode because of its larger gamut therefore giving them the option in the range of colours to 
use in their works before converting to the destination profile (CMYK). Again, there was the unravelling of the fact that some printers and designers stick to or are familiar with the primary CMYK and RGB colour spaces because they had no knowledge, for instance, of sRGB, Adobe RGB 98 among others.

The relationship between the platesetter resolution (dpi) and the screen ruling (lpi) determines the halftone quality of the printed output. The screen ruling used for a job depends on the resolution of the platesetter, as well as, the paper stock and type of press to be used to print the publication(Lawler, 2005).

Ascertaining the resolution chosen for coated and uncoated stock with a range between 100dpi- $300 \mathrm{dpi}$ and 72 dpi - 100 dpi respectively was given to respondents to choose for each stock they print on.

Coated stock and uncoated stock all have different ranges of resolutions they can handle. A rage of $72 \mathrm{dpi}-100 \mathrm{dpi}$ is fundamentally used for uncoated stock of paper. Between 100dpi - $300 \mathrm{dpi}$ is chosen for a coated stock of paper in the press house. Lawler (2005), advises that full-colour newspapers (uncoated stock) should be commonly printed using a screen ruling of 100 lpi because newsprint absorbs a great deal of ink and the presses operate at high speed. A greater response from the study brought to light the lack of attention placed on the resolution chosen for different stock of paper. Majority of presses choose between 100dpi - 300dpi for their resolution on uncoated stock. This choice has the tendency to oversaturate the uncoated paper stock and make the images look muddy and paper soaked as expressed by some of the operators. In addition, the grammage of the paper in question and its special characteristics enabled some presses to choose higher resolution for their uncoated stocks. Also, full-colour magazines on coated paper are usually printed with a screen ruling of $133 \mathrm{lpi}$, whereas sheet-fed and commercial printing is typically done at $150 \mathrm{lpi}($ Lawler, 2005). With no particular attention given to resolution, a greater percentage of the respondents chose resolution between $100-300$ dpi for their coated stocks jobs. The essence of resolution to them was not a great factor to be considered. To them, resolution selection is based on the type of job and the machine to be used for the printing.

ICC-Profiling has afforded operators with enough options in the form of rendering intents. The rendering intents are basically four - Relative Colorimetric, Absolute Colorimetric, Perceptual and Saturation. Each of these rendering intents have their type of graphic images they are applied to and is also in part based on the preference of the operator. This came to most pressmen as a new thing. They had seen it in dialogue boxes but had never known the essence of it. In the survey to know which one they normally use, Relative Colorimetric had the highest response rate because by observation that was the default setting and many of the respondents had never changed it. The other rendering intents had their fair share of being chosen but again were not chosen on purpose.

\section{Conclusion}

The aim of colour management is to ensure consistency, repeatability and predictability in coloured works (Ashe, 2014). This study has shown that there is little or no knowledge regarding the implement of colour management systems as means of ensuring print quality in many Ghanaian press houses especially in Accra and Kumasi. Many of the technicians that man these establishments have minimal knowledge of and effect of colour management, leading to the lack of calibration and profiling of equipment, the little or unavailability of colour measuring instruments and the over reliance of human visualisation for calibration where necessary. The study has also revealed the lack of standards in the Ghanaian printing environment, as a result, there is no strict competition. This probably has contributed to the outsourcing of printing jobs to companies in far east (India, Malaysia, China and Singapore) and South Africa. The study has shown that many press houses rely on the default settings of their printing equipment which sometimes affect the quality of print they produce.

To stem the problems associated with outsourcing of printing jobs, Ghanaian press houses should invest in strengthen their colour management systems, by purchasing the needed instruments to monitor the performance of their equipment as well as providing in-service training for their personnel to improve their knowledge base regarding issues in the printing industry.

\section{References}

i. Adams II, R. M., \& Weisberg, J. B. (2000). GATF Practical Guide to Color Management: GATFPress.

ii. Agfa-Gavaert, N. V. (1997). The secrets of color management: Digital color prepress (Vol. 5): Agfa Prepress Educational Publishing.

iii. Ashe, T. (2014). Color management \& quality output: Working with color from camera to display to print: Routledge.

iv. Enoksson, E. (2010). Toward better image reproduction in offset. KTH,

v. Green, P. (2010). Color management: understanding and using ICC profiles/edited by Phil Green. In: John Wiley and Sons.

vi. Köse, E., Şahinbașkan, T., \& Güler, İ. (2009). The investigation of effects of digital proofing systems used in colour management on print quality with neural networks. Expert Systems with Applications, 36(1), 745-754.

vii. Kotler, P., Armstrong, G., Harris, L., \& Piercy, N. (2013). Principles of Marketing (6th European ed.). Essex: Pearson Education Limited.

viii. Lawler, B. P. (2005). Official Adobe Print Publishing Guide: The Essential Resource for Design, Production, and Prepress, The: Adobe Press.

ix. Marin, J. (2004). Pain Of Color Management: A 2004 Satisfaction Survey: Graphic Arts Tech Fo.

x. Mejtoft, T. (2006). Strategies for Successful Digital Printing. Journal of Media Business Studies, 3(1), 53-74. 
xi. Nussbaum, P. (2010). Colour measurement and print quality assessment in a colour managed printing workflow. Faculty of Computer Science Media Technology Gjøvik University College, Norway: The Norwegian Color Research Laboratory

xii. Ploumidis, D. (2008). Quality control and the role of color management. Part I Color Management Series. Retrieved from https://flexoglobal.com/flexomag/08-May/flexomag-ploumidis.htm

xiii. Prempeh, E. G. (2011). Evaluation of the printing technology option of the Publishing Studies Programme in relation to the demands of the printing industry in Ghana. Retrieved from Kumasi:

xiv. Upton, S. (2001). How often should monitors be calibrated? Retrieved from http://www.chromix.com/colorsmarts/smartNote.cxsa?snid=472\&session=SessID:817A10931d91f0B0B4MUN26FB78C

xv. Waite, J. (2006). What to teach graphic design students about printing industry guidelines. Visual Communications Journal(1).

xvi. X-Rite, I. (1998). The Color Guide and Glossary:Communication, Measurement, and Control for Digital Imaging and Graphic Arts. Grandville, Mich.: X-Rite, Incorporated. 